\title{
ON THE COMPOSITUM OF TWO POWER SERIES RINGS
}

\author{
SHREERAM S. ABHYANKAR, WILLIAM HEINZER, AND SYLVIA WIEGAND
}

(Communicated by Louis J. Ratliff, Jr.)

ABSTRACT. This paper concerns subrings of the bivariate power series ring over a field.

In this note, we study subrings of the power series ring $k[[X, Y]]$ in two variables over a field $k$.

In particular, in $\S 1$, we exhibit an element $z$ in $k[[X, Y]]$ which is transcendental over the compositum $A=k[k[[X]], k[[Y]]]$ of the power series rings $k[[X]]$ and $k[[Y]]$. In fact we show that the power series ring $k[[X, Y]]$ has uncountable transcendence degree over the compositum $A$.

Stephen McAdam raised a question, discussed in [HW], concerning the existence of a non-Henselian Noetherian two-dimensional local domain $S$ such that $S / P$ is Henselian for each height one prime $P$. In $\S 2$ of this article, we construct such a domain $S$, lying between $k[[X, Y]]$ and the compositum $A$, and containing an element transcendental over $A$, such as $z$.

In $\S 3$, some questions are raised concerning various subrings of $k[[X, Y]]$. Also we include an argument due to Kunz that the compositum is not Noetherian.

We would like to thank Ray Heitmann for a careful reading of an earlier draft of this paper and for providing us with an improved proof (given in §1) of the transcendence of the element $z$ mentioned above. Also Heitmann has communicated to us another proof that the compositum $A$ is not Noetherian. We thank the referee for pointing out that the transcendence degree of $k[[X, Y]]$ over $A$ is uncountable.

\section{POWER SERIES}

Proposition 1.1. For every element a of the compositum $A=k[k[[X]], k[[Y]]]$, there exists a finite dimensional $k$-vector subspace $W$ of $k[[Y]]$ such that a $\in$ $W[[X]]$.

Received by the editors March 15, 1990 and, in revised form, June 21, 1990; presented June 1, 1990 at the Shreeram S. Abhyankar's Sixtieth Birthday Conference on Algebraic Geometry and Applications, sponsored by Purdue University and the National Science Foundation.

1980 Mathematics Subject Classification (1985 Revision). Primary 13J15, 13J05; Secondary 13B35, 13E05, 13A17.

Key words and phrases. Completion, Henselian, Noetherian, power series, transcendental.

S. Abhyankar and W. Heinzer were supported by the National Science Foundation. 
Proof. We see that $a$ can be expressed as a finite linear combination

$$
a=p_{1}(X) q_{1}(Y)+\cdots+p_{d}(X) q_{d}(Y),
$$

where each $p_{e}(X) \in k[[X]]$ and each $q_{e}(Y) \in k[[Y]]$. Because $a \in k[[Y]][[X]]$, we get

$$
a=r_{0}(Y)+r_{1}(Y) X+\cdots+r_{j}(Y) X^{j}+\cdots, \quad \text { with } r_{j}(Y) \in k[[Y]],
$$

and now upon letting

$$
p_{e}(X)=p_{e 0}+p_{e 1} X+\cdots+p_{e j} X^{j}+\cdots, \quad \text { with } p_{e j} \in k
$$

we obtain

$$
r_{j}(Y)=p_{1 j} q_{1}(Y)+\cdots+p_{d j} q_{d}(Y) \quad \text { with } p_{e j} \in k \text { for all } j
$$

and hence

$$
r_{j}(Y) \in W \text { for all } j
$$

where $W=$ the finite dimensional $k$-vector space generated by $q_{1}(Y), \ldots$, $q_{d}(Y)$. Thus $a \in W[[X]]$.

For convenience, we assign letters to some power series rings.

Notation 1.2. List of power series rings and localizations:

$$
\begin{aligned}
R & =k[[X, Y]] . \\
A & =k[k[[X]], k[[Y]]]=k[[X]][k[[Y]]]=k[[Y]][k[[X]]] . \\
D & =k[[Y]][X] . \\
E & =\bigcup_{W \in \mathscr{W}} k[W][[X]], \text { where } \mathscr{W} \text { is the set of all finite dimensional }
\end{aligned}
$$

$k$-vector spaces contained in $k[[Y]]$.

$$
\begin{aligned}
E_{0} & =\bigcup_{W \in \mathscr{W}} W[[X]] \\
& =\{\text { power series in } X, \text { with coefficients in some } W \in \mathscr{W}\} .
\end{aligned}
$$

Let $M$ denote the maximal ideal of $R$ and let $A^{\prime}=A_{A \cap M}$.

Corollary 1.3. (i) $D \subset A \subset E_{0} \subset E \subset R$.

(ii) None of these elements are in $A$ :

$$
\begin{aligned}
& u_{1}=\Sigma X^{i} Y^{i}, \\
& u_{2}=\Sigma X^{i}\left(Y^{i}+Y^{i+1}+\cdots\right), \\
& u_{3}=\Sigma X^{i} Y^{i !}, \\
& u_{4}=(1-X Y)^{1 / 2} .
\end{aligned}
$$

(All powers of $Y$ occur as coefficients of the powers of $X$ in $u_{4}$ ). Note that $u_{1}$ and $u_{3}$ are in $E \backslash E_{0}$, while $u_{2}$ is outside $E$.

Remarks 1.4. 1. Actually $u_{1}=1 /(1-X Y)$ and $u_{2}=u_{1} /(1-Y)$. Hence $u_{1}$ and $u_{2} \in A^{\prime}$. Question: are $u_{3}$ and $u_{4} \in A^{\prime}$ ? Is $u_{3}$ transcendental over $A$ ? 
2. It is possible to choose an uncountable set $\left\{f_{\alpha}(Y)\right\}_{\alpha \in \Omega}$ of algebraically independent power series in $Y$ over $k$. (This can be proved by a cardinality argument: it is true for the prime field $K$ of $k$, since $K$ is countable, but $K[[Y]]$ is uncountable and so has uncountable transcendence degree over $K$. It follows that the same holds for $k[[Y]]$.)

3. In [A1], it is shown that if $\left\{f_{\tau}(Y)\right\}_{\tau \in \Gamma}$ is a countably infinite set of algebraically independent power series in $Y$ over $k$, then $\left\{f_{\tau}(Y) X\right\}_{\tau \in \Gamma}$ is a countably infinite set of analytically independent power series over $k$.

Definition 1.5. Let $\Gamma$ be a countably infinite subset of $\Omega$ from Remark 1.4 (2), then $\left\{f_{\gamma}(Y)\right\}_{\gamma \in \Gamma}$ is a countably infinite set of algebraically independent power series over $k$. Set

$$
z=\sum_{\gamma \in \Gamma} f_{\gamma}(Y) X^{\gamma} \in R
$$

Proposition 1.6. The element $z$ from Definition 1.5 is transcendental over $F$, the quotient field of $A$.

Proof. We will omit the proof, since it is clearly contained in the proof of 1.8 below.

By revising the procedure used for construction of $z$, we can construct uncountably many transcendental elements $z_{\beta}$ as follows:

Definition 1.7. First, let $\left\{I_{\beta}: \beta \in \Theta\right\}$ be an uncountable disjoint collection of countably infinite subsets of $\Omega$. Say

$$
I_{\beta}=\{\beta(0), \beta(1), \ldots, \beta(n), \ldots\} .
$$

Let $\left\{f_{\alpha}(Y)\right\}_{\alpha \in \Omega}$, be an uncountable set of power series in $k[[Y]]$ which are algebraically independent over $k$. Now set

$$
z_{\beta}=\sum_{n=0}^{\infty} f_{\beta(n)}(Y) X^{n}, \quad \text { for each } \beta .
$$

Proposition 1.8. The elements $z_{\beta}$ are algebraically independent over the quotient field of $E$, and hence the power series ring $K[[X, Y]]$ has uncountable transcendence degree over $F$, the quotient field of $A$.

Proof. It will suffice to prove the following:

Claim. For each $\lambda \in \Theta, z_{\lambda}$ is transcendental over the quotient field of $E\left[\left\{z_{\beta}\right\}_{\beta \neq \lambda}\right]$. Proof of Claim. Suppose not. Write $z=z_{\lambda}$. Then there exists a finite subset $\left\{z_{1}, \ldots, z_{m}\right\}$ of $\left\{z_{\beta}\right\}_{\beta \neq \lambda}$ and a nontrivial expression

$$
a_{n} z^{n}+\cdots+a_{0}=0,
$$

where $a_{j} \in E\left[\left\{z_{1}, \ldots, z_{m}\right\}\right]$ for $0 \leq j \leq n$. By Proposition 1.1, there exists a finite-dimensional $k$-vector subspace $W$ of $k[[Y]]$ so that $a_{j} \in k[W]$ $\left[\left\{z_{1}, \ldots, z_{m}\right\}\right][[X]]$, for $0 \leq j \leq n$. Relabel $z_{l}=\sum_{i=0}^{\infty} f_{l i}(Y) X^{i}$, for $1 \leq$ $l \leq m$, and $z=\sum_{i=0}^{\infty} f_{i}(Y) X^{i}$. Then $a_{j} \in k[W]\left[\bigcup_{l=1}^{m}\left\{f_{l i}(Y)\right\}_{i=0}^{\infty}\right][[X]]$, for 
$0 \leq j \leq n$. Recall that the set $\left\{f_{i}(Y)\right\}_{i=0}^{\infty} \cup \bigcup_{l=1}^{m}\left\{f_{l i}(Y)\right\}_{i=0}^{\infty}$ is algebraically independent over $k$. Consequently, if $L$ denotes the algebraic closure of the quotient field of $k[W]\left[\bigcup_{l=1}^{m}\left\{f_{l i}(Y)\right\}_{i=0}^{\infty}\right]$ (in a fixed algebraic closure of $k[[Y, X]]$ ), then at most finitely many of the $f_{i}(Y)$ are in $L$.

Choose $i$ minimal so that $f_{i} \notin L$. Then we revise $z$ to be $\left(z-\sum_{t=0}^{i-1} f_{t}(Y) X^{t}\right)$ $/ X^{i}$, and revise the $a_{j}$ as necessary, including if some $s$ is such that $X^{s}$ divides $a_{j}$ for all $0 \leq j \leq n$, then we replace $a_{j}$ by $a_{j} / X^{s}$. As a result, we may suppose in $(*)$ that $a_{j} \in L[[X]]$ for all $j, z(Y, 0)=f_{0}(Y) \notin L$, and $a_{j}(Y, 0) \neq 0$, for some $j$. However, setting $X=0$ in $(*)$ :

$$
a_{n}(Y, 0) f_{0}^{n}+\cdots+a_{j}(Y, 0) f_{0}^{j}+\cdots+a_{0}(Y, 0)=0,
$$

leads to a nontrivial algebraic expression for $f_{0}(Y)$ over $L$, which is impossible since $L$ was algebraically closed.

Remarks 1.9. 1. Abhyankar and Moh have shown there exists an element of $k[[X, Y]]$ which is analytically independent over $k[[X, X Y]]$ [AM, Theorem 3]. Also Sheldon showed $k[[X, Y]]$ has infinite transcendence degree over the quotient field of $k[[X, X Y]]$ [Sh].

2. In his thesis $[\mathrm{H}]$, Huang did some interesting work on the algebraic closure of the quotient field $N((Y))$ of a power series ring $N[[Y]]$, where $N$ is an algebraically closed field. Newton's theorem [A2] or [C] states that, in case $N$ is an algebraically closed field of characteristic zero, the algebraic closure of $N((Y))$ is $N\langle Y\rangle_{b}=$ \{"power series" $\zeta$ in $Y$ over $N$ so that the exponents in $\zeta$ are a well-ordered subset of the rationals and the set of denominators occurring in the exponents of $\zeta$ is bounded $\}=\bigcup\left\{N\left(\left(Y^{1 / n}\right)\right): n\right.$ a positive integer\}.

However for characteristic $p \neq 0$, Chevalley shows $N\langle Y\rangle_{b}$ is smaller that the algebraic closure by exhibiting a specific irreducible polynomial over $N\langle Y\rangle_{b}$ of degree $p$ [C, p. 64]. In [A1], a factorization of this polynomial is given, with the factors being generalized power series where the exponents have unbounded denominator. Using this type of factorization, M. F. Huang (in $[\mathrm{H}]$ ), shows that, if $N$ is an algebraically closed field of characteristic $p>0$, then $N\langle Y\rangle_{b, p}$ contains an algebraic closure of $N((Y))$. Here, $N\langle Y\rangle_{b, p}=$ \{ "power series" $\zeta$ in $Y$ over $N$ so that the exponents in $\zeta$ are a well-ordered subset of the rationals and the set of denominators occurring in the exponents of $\zeta$ is bounded except that these denominators may include arbitrarily large powers of $p$ \}. (Huang also gives criteria for certain generalized power series to be algebraic over the field of meromorphic functions $N((Y))$. For example, if $a_{i}$ are integers between 0 and $p-1$ and $\bar{a}_{i}$ denotes the image of $a_{i}$ in the field $N$, then Huang shows that $\alpha=\sum_{i=1}^{\infty} \bar{a}_{i} Y^{-1 / p^{i}}$ is algebraic over $N((Y))$ if and only if $\beta=\sum_{i=1}^{\infty} a_{i} / p^{i}$ is a rational number.) 


\section{A NON-HENSELIAN EXAMPLE}

Proposition 2.1. Let $(\mathscr{R}, \mathscr{M})$ be an analytically normal, local, unique factorization domain with completion $(\widehat{\mathscr{R}}, \widehat{\mathscr{M}})$. Assume that for each nonzero prime $\mathscr{Q}$ of $\widehat{\mathscr{R}}, \mathscr{Q} \cap \mathscr{R} \neq(0)$, and that for all but at most a finite number of the height one primes $\mathscr{P}$ of $\mathscr{R}, \mathscr{R} / \mathscr{P}$ is complete. Let $\mathscr{K}$ be the field of fractions of $\mathscr{R}$, and $\widehat{\mathscr{K}}$ the field of fractions of $\widehat{\mathscr{R}}$. If $\mathscr{L}$ is any field between $\mathscr{K}$ and $\widehat{\mathscr{K}}$, then $\mathscr{S}=\mathscr{L} \cap \widehat{\mathscr{R}}$ is a normal local domain with maximal ideal $\widehat{\mathscr{M}} \cap \mathscr{S}$. Moreover, $\operatorname{dim} \mathscr{R}=\operatorname{dim} \mathscr{S}$.

Proof. It is always true that $\mathscr{S}$ is a Krull domain $(\mathscr{S}$ is an intersection of the discrete valuation rings formed when the essential valuations of the Krull domain $\widehat{\mathscr{R}}$ are restricted to $\mathscr{L}$ ) and so $\mathscr{S}$ is normal [N, 33.4]. Also each height one prime in $\mathscr{S}$ is the contraction of a height one prime in $\widehat{\mathscr{R}}$. In view of the theorem of Mori-Nishimura [M, Theorem 104], to show that $\mathscr{S}$ is Noetherian, it suffices to show that each prime ideal $\mathscr{Q}$ in $\mathscr{S}$ of height greater than 1 is finitely generated. If $\mathscr{P}$ is a height one prime in $\mathscr{R}$ such that $\mathscr{R} / \mathscr{P}$ is complete, then

$$
\mathscr{R} / \mathscr{P}=\widehat{\mathscr{R}} / \mathscr{P} \widehat{\mathscr{R}},
$$

so $\mathscr{P} \widehat{\mathscr{R}}$ is a height one prime in $\widehat{\mathscr{R}}$. Our hypothesis implies that all but a finite number of the height one primes of $\widehat{\mathscr{R}}$ are of the form $\mathscr{P} \widehat{\mathscr{R}}$ for $\mathscr{P}$ a height one prime of $\mathscr{R}$. Since $\mathscr{Q}$ is a prime of height greater than 1 in $\mathscr{S}=\widehat{\mathscr{R}} \cap \mathscr{L}$ and $\mathscr{Q}$ is the union of the height one primes of $\mathscr{S}$ contained in $\mathscr{Q}$, all of which are contractions of height one primes of $\mathscr{\mathscr { R }}$, there exists a height one prime $\mathscr{P}$ of $\mathscr{R}$ such that $\mathscr{R} / \mathscr{P}$ is complete and $\mathscr{P} \mathscr{R} \cap \mathscr{S}$ is contained in $\mathscr{Q}$. Therefore

$$
\mathscr{R} / \mathscr{P}=\mathscr{S} /(\mathscr{P} \widehat{\mathscr{R}} \cap \mathscr{S})=\widehat{\mathscr{R}} / \widehat{\mathscr{P}} \text {. }
$$

Since $\mathscr{R}$ is a unique factorization domain, $\mathscr{P}$ is principal. Now, since principal ideals in a Krull domain have no imbedded primes,

$$
\mathscr{P} \widehat{\mathscr{R}} \cap \mathscr{S}=\mathscr{P} \mathscr{S} \text {. }
$$

Thus $\mathscr{S} / \mathscr{P} \mathscr{S}$ is Noetherian and so $\mathscr{Q} / \mathscr{P} \mathscr{S}$ is finitely generated. Hence $\mathscr{Q}$ is finitely generated, which completes the proof that $\mathscr{S}$ is Noetherian. Now clearly $\mathscr{S}$ is local, with maximal ideal $\widehat{\mathscr{M}} \cap \mathscr{S}$. Moreover, the existence of a principal height one prime $\mathscr{P}$ of $\mathscr{R}$ such that $\mathscr{R} / \mathscr{P}=\mathscr{S} / \mathscr{P} \mathscr{S}$ implies that $\operatorname{dim} \mathscr{R}=\operatorname{dim} \mathscr{S}$ [M, Proposition 12K, p. 78].

By Proposition 1.6, there exists an element $z$ in $R=k[[X, Y]]$ which is transcendental over $F$, where $F$ is the field of fractions of $A$. Let

$$
S=R \cap F(z) .
$$

Proposition 2.2. $S$ is not Henselian, but $S / P$ is Henselian, for each height one prime $P$ of $S$.

Proof. Notice that $D^{\prime}=D_{M \cap D}$, where $D=k[[Y]][X]$, satisfies the hypotheses on $\mathscr{R}$ in Proposition 2.1, and thus $S$ is a two dimensional normal local do- 
main. The fact that $S$ is not Henselian follows from a result that goes back to F. K. Schmidt [S], [BBKN, Satz 2.3.11, p. 60], which states that any rank-one discrete valuation domain of the field of fractions of a Henselian domain must contain the domain. Therefore the field of fractions of a Henselian domain which is not a field cannot be a function field of positive transcendence degree over some subfield. It follows that $S$, which has field of fractions $F(z)$, is not Henselian. Now also for each height one prime $P$ of $S$,

$$
S / P \approx R / P R \approx A /(P \cap A),
$$

so $S / P$ is Henselian, for each height one prime $P$ of $S$.

\section{SOME FURTHER REMARKS AND QUESTIONS}

It would be interesting to know more about the structure and quotient fields of the power series rings discussed in $\S \S 1$ and 2 , so that, for example, we could more easily decide if a given power series belongs to them.

In addition, we are interested in the following power series rings:

$B=k[k[[X]], k[[Y]], k[[X+Y]]]$. (Notes: $\Sigma(X+Y)^{i} \notin A$, but it is in $F$. Is the quotient field of $B$ properly larger than $F$ ? $\Sigma(X+Y)^{i !} \notin A$; is it in $F$ ?)

$B_{\Omega}=k[\{k[[X+a Y]], a \in \Omega\}]$, for $\Omega$ any subset of $k \cup\{\infty\}$. Convention: For $a=\infty, X+a Y=Y$. Also $B_{1}$ denotes $k[k[[X+Y]]]=k[[X+Y]]$, etc. (Conjecture: If $\Omega \neq \Omega^{\prime}$, then $B_{\Omega} \neq B_{\Omega^{\prime}}$.)

$C_{\Omega}=k[\{k[[f]]: f \in \Omega\}]$, where $\Omega$ is a subset of the polynomials in $X$ and $Y$ with zero constant term. Two specific rings here that it would be interesting to consider are $C_{\Omega_{1}}$ and $C_{\Omega_{2}}$, where $\Omega_{1}$ is the set of irreducible polynomials in $X$ and $Y$ with zero constant term, and $\Omega_{2}$ is the set of all polynomials in $X$ and $Y$ with zero constant term.

Also we wonder which of the rings mentioned in this paper (and/or the appropriate localizations) are Noetherian, Henselian, complete, unique factorization domains, unicomplete, or unihenselian? (We say a ring $S$ is uni- $P$ for a property $P$, if $S / H$ is $P$ for every height one prime ideal $H$ in $S$.) What are their prime spectra like?

The following result concerning the Noetherian question was communicated to us by Reinhold Hübl and Ernst Kunz. (Kunz stated that he believes it is well-known. We are grateful to Kunz for sending us the proof so that we could include it here.)

Proposition 3.1. Let $k \subseteq K$ be fields such that $K$ has infinite transcendence degree over $k$. Then $K \otimes_{k} K$ is not Noetherian.

It follows that $k[[X]] \otimes_{k} k[[X]]$, which localizes to $k((X)) \otimes_{k} k((X))$, is not Noetherian. In other words, the compositum $A$, which is isomorphic to $k[[X]] \otimes_{k} k[[Y]]$, is not Noetherian.

Proof (due to Kunz). Choose a transcendence basis $\left\{x_{\lambda}\right\}$ of $K$ over $k$. Then $K \otimes_{k} K$ has a free basis over $k\left(\left\{x_{\lambda}\right\}\right) \otimes_{k} k\left(\left\{x_{\lambda}\right\}\right)$, and 1 may be taken as part of the free basis. Therefore if $K \otimes_{k} K$ were Noetherian, it would follow 
that $k\left(\left\{x_{\lambda}\right\}\right) \otimes_{k} k\left(\left\{x_{\lambda}\right\}\right)$ would also be Noetherian (see, for example, [AH]). Now the module of differentials $\Omega_{k\left(\left\{x_{\lambda}\right\}\right) / k}^{1}$ is infinite dimensional as a $k\left(\left\{x_{\lambda}\right\}\right)$ vector space $[\mathrm{K}, 5.4]$. This implies $\Omega_{k\left[\left\{x_{\lambda}\right\}\right] / k}^{1}$ cannot be finitely generated as a $k\left[\left\{x_{\lambda}\right\}\right]$-module, since

$$
\Omega_{k\left(\left\{x_{\lambda}\right\}\right) / k}^{1}=k\left(\left\{x_{\lambda}\right\}\right) \otimes_{k\left[\left\{x_{\lambda}\right\}\right]} \Omega_{k\left[\left\{x_{\lambda}\right\}\right] / k}^{1}
$$

[K, 4.22]. But $\Omega_{k\left[\left\{x_{\lambda}\right\}\right] / k}^{1} \cong I / I^{2}$ where $I$ is the kernel of the map

$$
k\left[\left\{x_{\lambda}\right\}\right] \otimes_{k} k\left[\left\{x_{\lambda}\right\}\right] \rightarrow k\left[\left\{x_{\lambda}\right\}\right] \quad(\text { via } a \otimes b \mapsto a b)
$$

by $[\mathrm{K}, 1.21]$. Then $I$ cannot be finitely generated as an ideal of $k\left[\left\{x_{\lambda}\right\}\right] \otimes_{k}$ $k\left[\left\{x_{\lambda}\right\}\right]$. Therefore $k\left[\left\{x_{\lambda}\right\}\right] \otimes_{k} k\left[\left\{x_{\lambda}\right\}\right]$ is not Noetherian and neither is $K \otimes_{k} K$.

Remark. (i) As a consequence of 3.1, Hübl also observed that the localization $A^{\prime}$ of $A=k[k[[X]], k[[Y]]]$ is not Noetherian. Since by Proposition 2.1, $R \cap F$ is Noetherian, it follows that $A^{\prime}$ is properly contained in $R \cap F$.

(ii) In an earlier version of the paper, we asked about the existence of some concrete power series which are transcendental over the ring $A$. Hübl gave us the following example: Choose a countably infinite set $\left\{\lambda_{i} \mid 0 \leq i<\infty\right\}$ of complex numbers which are linearly independent over the rational numbers, then the power series $\left\{e^{\lambda_{i} Y} \mid i \in I\right\}$ are algebraically independent over the complex numbers. (For example take $\lambda_{i}=\sqrt[(i+1)]{2}$.) Now, as in Definition 1.5, set $z=\sum_{i=0}^{\infty} e^{\lambda_{i} Y} X^{i}$. By Proposition 1.9, $z$ is transcendental over $A$.

Added in proof. Rayner has also described an algebraically closed field containing a power series ring $N[[Y]]$, where $N$ is an algebraically closed field of characteristic $p \neq 0[\mathrm{R}]$.

\section{REFERENCES}

[A1] S. Abhyankar, Two notes on formal power series, Proc. Amer. Math. Soc. 7 (1956), 903-905.

[A2] - Algebraic geometry for scientists and engineers, Surveys and Monographs of the Amer. Math. Soc., no. 35, Amer. Math. Soc., Providence, RI, 1990.

[AH] S. Abhyankar and W. Heinzer, Integral closure and ramification of prime ideals in infinite algebraic field extensions, J. Algebra (to appear).

[AM] S. Abhyankar and T. Moh, On analytic independence, Trans. Amer. Math. Soc. 219 (1976), 77-87.

[BKKN] R. Berger, R. Kiehl, E. Kunz, and J.-J. Nastold, Differentialrechnung In Der Analytischen Geometrie, Lecture Notes in Math., vol. 38, Springer-Verlag, New York, 1967.

[C] C. Chevalley, Introduction to the theory of algebraic functions of one variable, Surveys and Monographs of the Amer. Math. Soc., no. 6, Amer. Math. Soc., Providence, RI, 1951.

[HW] W. Heinzer and S. Wiegand, Prime ideals in two-dimensional polynomial rings, Proc. Amer. Math. Soc. 107 (1989), 577-586.

[H] M. F. Huang, On the algebraic closure of the field of meromorphic functions over an algebraically closed field of characteristic, Ph.D. Thesis, Purdue University, 1968.

[K] E. Kunz, Kähler differentials, Advanced Lectures in Mathematics, Friedr. Viewig \& Sohn, Braunschweig, 1986. 
[M] H. Matsumura, Commutative algebra, Benjamin/Cummings, Reading, MA, 1980.

[N] M. Nagata, Local rings, Interscience, New York, 1962.

[R] F. J. Rayner,, An algebraically closed field, Glasgow Math. J. 9 (1968), 146-151.

[S] F. K. Schmidt, Mehrfach perfekte Korper, Math. Ann. 108 (1933), 1-25.

[Sh] P. B. Sheldon, How changing $D[[x]]$ changes its quotient field, Trans. Amer. Math. Soc. 159 (1971), 223-244.

Department of Mathematics, Purdue University, West Lafayette, Indiana 47907

(Sylvia Wiegand) Department of Mathematics, University of Nebraska, Lincoln, NEBRASKA 68588-0323 